\section{(1) \\ CrossMark}

\title{
Detecting lung infections in breathprints: empty promise or next generation diagnosis of infections
}

\author{
Hossam Haick ${ }^{1}$ and Sylvia Cohen-Kaminsky ${ }^{2,3,4,5}$
}

Affiliations: ${ }^{1}$ Dept of Chemical Engineering and Russell Berrie Nanotechnology Institute, Technion - Israel Institute of Technology, Haifa, Israel. ${ }^{2}$ INSERM UMR-S 999, Hypertension Artérielle Pulmonaire, Physiopathologie et Innovation Thérapeutique, LabEx LERMIT, Le Plessis-Robinson, France. ${ }^{3}$ Université ParisSud, Faculté de Médecine, Le Kremlin-Bicêtre, France. ${ }^{4}$ Centre Chirurgical Marie Lannelongue, Département de Recherche Médicale, Le Plessis-Robinson, France. ${ }^{5} \mathrm{AP}-\mathrm{HP}, \mathrm{DHU}$ TORINO, Centre National de Référence de l'Hypertension Pulmonaire Sévère, Hôpital Bicêtre, Le Kremlin-Bicêtre, France.

Correspondence: Hossam Haick, Dept of Chemical Engineering and Russell Berrie Nanotechnology Institute, Technion - Israel Institute of Technology, Haifa 3200003, Israel. E-mail: hhossamatechnion.ac.il

@ERSpublications

A discussion of evidence on the link between breathprints of bacterial lung infections and the immune response http://ow.ly/DgOOb

A wide spectrum of diagnostic technologies and tools are used to identify the agents causing infectious diseases [1-3]. It is increasingly recognised that an improved diagnostic tool should evolve into a personalised approach, fully taking into account 1) identification of individuals at risk of developing diseases; 2) interpretation of diagnostic tests; 3) providing prognostic information; and 4) predicting and following the efficacy of therapies [4]. A new noninvasive and potentially inexpensive frontier in the diagnosis of infectious diseases relies on the detection of volatile organic compounds (VOCs), which are organic compounds that have a high vapour pressure in ordinary room-temperature conditions, from exhaled breath [5-8].

Impressive empirical data have confirmed the potential of these compounds to serve as a basis for a noninvasive, simple, inexpensive and easy-to-use diagnostic tool [5-19]. In fact, monitoring VOCs in the breath may soon become an interesting supplement (or even an alternative) to conventional medical diagnostics, thanks to the rapid advances in the techniques for breath collection and gas-analysis [5-19]. This novel approach could revolutionise infectious disease care and management by allowing noninvasive in vivo differential diagnosis, in vitro prediction of the potential progression of infected cells, tailoring of individual treatment and real-time monitoring of therapeutic success [20-24]. Still, breath analysis is a very young "omic" field of research and faces challenges, mainly because the biochemical mechanisms behind the disease-related VOCs are largely unknown $[5,8]$.

Recently, several studies have speculated that the distinguishing features of each breathprint do not arise solely from pathogen metabolism, but are also due to changes of host VOCs, possibly in conjunction with the immunological response [25, 26]. However, the relationship between these three aspects has been lacking direct experimental validation. In this issue of the European Respiratory Journal, BEAN et al. [27] provide a tentative exploration of the mutual relationships between the above mentioned factors by exposing mouse airways to the lysates of two clinically important opportunistic pathogens (Pseudomonas aeruginosa and Staphylococcus aureus) to obtain breathprints without metabolic contributions from living bacterial cells. The lysates activated the host's immune response, which the authors hypothesised would generate pathogen-specific changes in the breathprint. Using secondary electrospray ionisation-mass spectrometry (SESI-MS), the authors found this was the case. Correlations between breathprint peaks and 
cytokine concentrations, and neutrophil and leukocyte counts in bronchoalveolar lavage fluid, provided evidence linking breath VOCs to host immunology. In addition, the authors found that breathprints of lysate exposure contain volatile signatures different from the breathprints of the corresponding active infections as well as from uninfected controls, and that they are predictive of the bacterial lysate source up to 5 days after the initial exposure. These results may have important implications for the detection of latent bacterial lung infections, for monitoring and managing the spread of respiratory diseases, and for the assessment and monitoring of new and existing bacterial vaccines.

To illustrate the impact of these results, we consider the case of tuberculosis. While there are several diagnostic methods for detection of active tuberculosis (e.g. sputum smear microscopy, tuberculosis culture from sputum and Xpert MTB/RIF [28]), and at the proof-of-concept stage a breath test using nanomaterial-based sensors [13], this is not the case for latent tuberculosis. The Mantoux tuberculin skin test [29] and interferon- $\gamma$ release assays are methods based on immunological tests and do not distinguish between active and latent disease. In these cases, a false-negative is entirely possible, even when multiple test modalities are employed, and no test currently tackles the active versus latent distinction. Recalling these limitations and considering the results reported by BEAN et al. [27], there is now a founding basis for how latent tuberculosis (or other infectious diseases) could be detected in an accurate and, importantly, noninvasive manner, namely from exhaled breath samples. However, it should be kept in mind that the immune response to live bacteria is likely to differ from the response to bacterial lysates. The authors have started to address this by assessing infections and their natural clearance over several days. Indeed, the breathprints from mice with live infections of $S$. aureus and $P$. aeruginosa contained features that differed from the lysate, i.e. nonmetabolising bacteria, experiments [30].

It is well known that pulmonary macrophages play a role in defence against respiratory infections and that they initiate anti-infective inflammation. One of the mechanisms involved in this process is phagocytosis of the pathogen, which induces the release of cytokines. Therefore, one could expect that the host contribution to the VOCs detected in breath following live pathogen infection will not be the same than those produced by the host after exposure to cell lysates. In addition, it is not obvious that the cytokines produced by the host in response to live bacteria and to bacterial lysates would be the same. Figure 1 shows a cartoon depicting the possible contributions to breathprints from infection and lysate exposure situations, and suggests that infection breathprints contain additional information even after bacterial clearance. Breathprints from individuals with infectious diseases will not be limited to the VOCs produced by bacteria and the host response to bacterial antigens. The host may also respond to the bacterial metabolites, which may include volatile metabolites and vice versa. Therefore the breathprint from a patient may provide a global view of these reactions. In this clinical context, however, it is not really important to know if the VOCs forming the breathprint signature come from: 1) the bacteria; 2) the host immune response to bacterial antigens; and/or from 3) the host response to bacterial products/metabolites. The collective sensing of the spectrum of VOCs and global analysis of the breath VOCs using sensor arrays is likely to give better sensitivity to detect infections, either acute or latent, and direct trials in humans with different infectious conditions are necessary to provide the proofs of concept and to assess the efficacy of any breathprint-based diagnostic tool $[5,6,12]$.

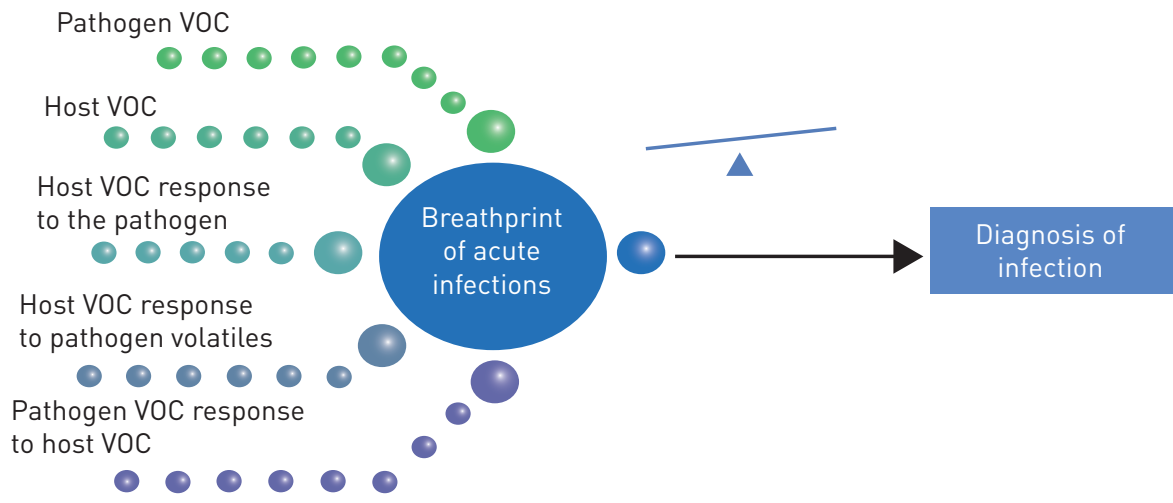

FIGURE 1 Potential contributions to breathprints from lung infections: the relative contributions of the host and the pathogen to the breathprint are intimately linked. In acute infection the breathprint is a balanced mixture of volatile organic compounds (VOCs) from the pathogen, VOCs from the host, VOCs from the host in response to the pathogen (which include VOCs linked to the immune response), VOCs from the host in response to pathogen VOCs and VOCs from the pathogen in response to host VOCs. 
For the reported results to become a clinical reality, several advances in the knowledge of specific volatile biomarkers and sensor development need to occur. For the delineation of the metabolic pathways leading to the generation of potential VOC biomarkers, future studies have to address the link between the mass-to-charge ratios reported in the paper and their chemical nature, via, for example, more sophisticated spectrometry techniques (such as two-dimensional gas chromatography-mass spectrometry) with larger databases of compounds than SESI-MS. Knowing the chemical structure of the reported mass-to-charge ratios (i.e. the mass divided by the charge number) would allow understanding the biochemical pathway of consumption and/or release of the reported VOCs and to put them in more specific, biochemical perspective. From a different angle, defining the exact structure based on the pre-evaluated breath VOCs will be helpful for designing and synthesising the recognition elements of chemical sensors for maximum sensitivity and selectivity or refining the range of chemical compounds in portable mass spectrometers. Regardless, to generate the broadest application to improve human health, a portable, easy-to-use, inexpensive device that would enable this technology at affordable prices is the goal $[5,6,9]$. The insight of BEAN et al. [27] into the origin of breath composition is the start of many steps toward that end-point.

\section{References}

1 World Health Organization (WHO). Global Status Report on Noncommunicable Diseases 2010. Geneva, WHO Press, 2011; pp. vii-ix.

2 World Health Organization (WHO). Communicable Disease Alert and Response for Mass Gatherings: Key Considerations. Geneva, WHO Press, 2008.

3 Kuhn M, Goebel W, Philpott DJ, et al. Overview of the bacterial pathogens. In: Kaufmann SHE, Sher A, Ahmed R, eds. Immunology of Infectious Diseases. Washington, ASM Press, 2002; pp. 5-23.

4 Karnon J, Goyder E, Tappenden P, et al. A review and critique of modelling in prioritising and designing screening programmes. Health Technol Assess 2007; 11: 1-145.

5 Haick H, Broza YY, Mochalski P, et al. Assessment, origin, and implementation of breath volatile cancer markers. Chem Soc Rev 2014; 43: 1423-1449.

6 Konvalina G, Haick H. Sensors for breath testing: from nanomaterials to comprehensive disease detection. Acc Chem Res 2014; 47: 66-76.

7 Amann A, Mochalski P, Ruzsanyi V, et al. Assessment of the exhalation kinetics of volatile cancer biomarkers based on their physicochemical properties. J Breath Res 2014; 8: 016003.

8 Hakim M, Broza YY, Barash O, et al. Volatile organic compounds of lung cancer and possible biochemical pathways. Chem Rev 2012; 112: 5949-5966.

9 Broza YY, Haick H. Nanomaterial-based sensors for detection of disease by volatile organic compounds. Nanomedicine (Lond.) 2013; 8: 785-806.

10 Barker M, Hengst M, Schmid J, et al. Volatile organic compounds in the exhaled breath of young patients with cystic fibrosis. Eur Respir J 2006; 27: 929-936.

11 Borrill ZL, Roy K, Singh D. Exhaled breath condensate biomarkers in COPD. Eur Respir J 2008; 32: 472-486.

12 Chapman EA, Thomas PS, Stone E, et al. A breath test for malignant mesothelioma using an electronic nose. Eur Respir J 2012; 40: 448-454.

13 Nakhleh MK, Jeries R, Gharra A, et al. Detecting active pulmonary tuberculosis with a breath test using nanomaterial-based sensors. Eur Respir J 2014; 43: 1522-1525.

14 Wheelock CE, Goss VM, Balgoma D, et al. Application of 'omics technologies to biomarker discovery in inflammatory lung diseases. Eur Respir J 2013; 42: 802-825.

15 Westhoff M, Litterst P, Maddula S, et al. Differentiation of chronic obstructive pulmonary disease (COPD) including lung cancer from healthy control group by breath analysis using ion mobility spectrometry. Eur Respir $J$ 2011; 38: Suppl. 55, 1699.

16 Becher G, Hillmann A, Purkhart R, et al. Adaptation of differential ion mobility spectrometry (DMS) for discrimination of specific biomarkers in exhaled breath in patients with severe renal-pulmonary dysfunction. Eur Respir J 2011; 38: Suppl. 55, 4041.

17 Xu ZQ, Broza YY, Ionsecu R, et al. A nanomaterial-based breath test for distinguishing gastric cancer from benign gastric conditions. Br J Cancer 2013; 108: 941-950.

18 Peng G, Hakim M, Broza YY, et al. Detection of lung, breast, colorectal, and prostate cancers from exhaled breath using a single array of nanosensors. Br J Cancer 2010; 103: 542-551.

19 Bos LD, Weda H, Wang Y, et al. Exhaled breath metabolomics as a noninvasive diagnostic tool for acute respiratory distress syndrome. Eur Respir J 2014; 44: 188-197.

20 Davies MPA, Barash O, Jeries R, et al. Unique volatolomic signatures of TP53 and KRAS in lung cells. Br J Cancer 2014; 111: 1213-1221.

21 Peled N, Barash O, Tisch U, et al. Volatile fingerprints of cancer specific genetic mutations. Nanomedicine 2013; 9: 758-766.

22 Barash O, Peled N, Tisch U, et al. Classification of lung cancer histology by gold nanoparticle sensors. Nanomedicine 2012; 8: 580-589.

23 Amal H, Ding L, Liu BB, et al. The scent fingerprint of hepatocarcinoma: in-vitro metastasis prediction with volatile organic compounds (VOCs). Int J Nanomedicine 2012; 7: 4135-4146.

24 Barash O, Peled N, Hirsch FR, et al. Sniffing the unique "odor print" of non-small-cell lung cancer with gold nanoparticles. Small 2009; 5: 2618-2624.

25 Aksenov AA, Gojova A, Zhao W, et al. Characterization of volatile organic compounds in human leukocyte antigen heterologous expression systems: a cell's "chemical odor fingerprint". Chembiochem 2012; 13: 1053-1059.

26 Zhu J, Bean H, Jiménez-Díaz J, et al. Secondary electrospray ionization-mass spectrometry (SESI-MS) breathprinting of multiple bacterial lung pathogens, a mouse model study. J Appl Physiol 2013; 114: 1544-1549. 
27 Bean HD, Jiménez-Díaz J, Zhu J, et al. Breathprints of model murine bacterial lung infections are linked with immune response. Eur Respir J 2015; 45: 181-190.

28 Weyer K, Mirzayev F, Migliori GB, et al. Rapid molecular TB diagnosis: evidence, policy making and global implementation of Xpert MTB/RIF. Eur Respir J 2013; 42: 252-271.

29 Yassin MA, Petrucci R, Garie KT, et al. Use of tuberculin skin test, IFN- $\gamma$ release assays and IFN- $\gamma$-induced protein-10 to identify children with TB infection. Eur Respir J 2013; 41: 644-648.

30 Zhu J, Jiménez-Díaz J, Bean HD, et al. Robust detection of $P$. aeruginosa and $S$. aureus acute lung infections by secondary electrospray ionization-mass spectrometry (SESI-MS) breathprinting: from initial infection to clearance. J Breath Res 2013; 7: 037106. 